\title{
MAGNETIC LEVITATION OF LARGE LIQUID VOLUME
}

\author{
V. Bojarevics, A. Roy, K.A. Pericleous \\ School of Computing and Mathematical Sciences, \\ University of Greenwich, London, England
}

\begin{abstract}
It is well known from experiments and industrial applications of cold crucible melting that an intense AC magnetic field can be used to levitate large volumes of liquid metal in the terrestrial conditions. The levitation confinement mechanism for large volumes of fluid is considerably different from the case of a small droplet, where surface tension plays a key role in constraining the liquid outflow at the critical bottom point. The dynamic interaction between the oscillatory motion of the free surface and the effects of turbulent flow is analysed using a unified numerical model, which describes the time dependent behaviour of the liquid metal and the magnetic field. The MHD modified $k-\omega$ turbulence model is used to describe the mixing and damping properties at smaller scales not resolved by the macro model. The numerical multiphysics simulations suggest that it is possible to levitate a few kilograms of liquid metal in a cold crucible without requiring mechanical support from the container walls. Possible applications to the processing of reactive metals are discussed.
\end{abstract}

Introduction. There is a growing number of applications, where an electrically conducting fluid volume is confined or even levitated using a high frequency AC magnetic field. A well known example is the non-contact measurement of material properties, particularly of liquid metals or silicon, which can be deduced from experimental observation of the thermal and surface oscillations in a levitated liquid sample (typically $1-10 \mathrm{~mm}$ in diameter) [1].

On a larger scale, there is a demand for melting reactive materials without contamination, for example, titanium alloys for high quality castings. However, it is often difficult to achieve the required superheat in the melt with traditional 'cold' crucible-type furnaces due to a partial contact with the water-cooled copper walls [2]. If the contact was avoided, thermal losses would be limited only by radiation and possible evaporation. This would produce a higher superheat and permit investigation of materials at extreme temperatures. It could allow large volumes of metal to be evaporated for coating purposes [3] or to supply superheated melts without contamination for metal powder production. At the other extreme, a highly under-cooled liquid can be obtained before solidification to a glassy structure in the levitated conditions in the absence of crystallization centres [4]. Cold crucible melting may also be used for element separation and concentration, for instance, in treating the nuclear fusion products generated after reprocessing of spent fuel [5]. Growing demands on metal cleanness and impurity control by size require a rapid analysis technique to determine the content of impurities in samples. Melting samples in the presence of an electromagnetic field can help to concentrate inclusions in specific positions on the surface [6].

Existing experimental evidence suggests that it is possible to melt and levitate several kilograms of liquid metal [7], but it is not explained satisfactorily from a theoretical point of view. A very challenging example of "semi-levitating" up to $500 \mathrm{~kg}$ of molten titanium is presented in a recent publication [8]. In the present work, we show by numerical simulation that it is possible, with optimised geom- 


\section{Bojarevics, A.Roy, K.A.Pericleous}

etry, to levitate $2 \mathrm{~kg}$ of liquid metal without allowing contact to occur with the cold crucible walls. Two independent numerical models: the commercial package COMSOL and the spectral-collocation based free surface code SPHINX [9] are used for this task.

SPHINX solves the transient electromagnetic, fluid flow and thermodynamic equations, which describe the dynamic behaviour of the levitated droplet. The model incorporates free surface deformation and follows the time evolution of the oscillations, which result from the interaction between the electromagnetic gravity forces and the turbulent momentum transport. The $k-\omega$ turbulence model was applied previously (with some modifications to account for the effect of magnetic damping) [11] to predict the cold crucible melting process with increased superheat in the presence of an additional DC magnetic field. The model produced results, which were confirmed experimentally [10]. COMSOL is used to investigate 3-dimensional features of the electromagnetic field associated with the finger segments, and to confirm the validity of an assumption used in the SPHINX analysis, namely, that the field seen by the levitated charge is approximately axisymmetric.

Numerical model. The SPHINX model is based on the numerical solution of the turbulent momentum and heat transfer equations for an incompressible fluid:

$$
\begin{aligned}
\partial_{t} \mathbf{v}+(\mathbf{v} \cdot \nabla) \mathbf{v} & =-\frac{1}{\rho} \nabla p+\nabla \cdot\left(\nu_{\mathrm{e}}\left(\nabla \mathbf{v}+\nabla \mathbf{v}^{\mathrm{T}}\right)\right)+\frac{1}{\rho} \mathbf{f}+\mathbf{g}, \\
\nabla \cdot \mathbf{v} & =0 \\
C_{p}\left(\partial_{t} T+\mathbf{v} \cdot \nabla T\right) & =\nabla \cdot\left(C_{p} \alpha_{\mathrm{e}} \nabla T\right)+\frac{1}{\rho \sigma}|\mathbf{J}|^{2},
\end{aligned}
$$

where $v c v$ is the velocity vector, $p$ - the pressure, $\rho$ - the density, $\nu_{\mathrm{e}}$ is the effective viscosity (which is computed from the time dependent 2-equation $k$ - $\omega$ model), $\mathbf{f}$ is the electromagnetic force, $\mathbf{g}$ - the gravity vector, $T$ - the temperature, $\alpha_{\mathrm{e}}$ is the effective thermal diffusivity (related to $\nu_{\mathrm{e}}$ ), $C_{p}$ is the specific heat, and $|\mathbf{J}|^{2} / 2$ is the Joule heat. Equations (1)-(3), which are solved in spherical coordinates, were used in previous publications [2], [9] to describe the flow in an axisymmetric droplet of fluid. The boundary conditions at the external free surface are stated for the hydrodynamic stress tensor $\boldsymbol{\Pi}$ component projections on the surface:

$$
\Pi_{\mathrm{nn}}=\gamma K, \quad \Pi_{\mathrm{n} \tau}=\gamma_{T}\left(\nabla T \cdot \mathbf{e}_{\mathrm{n} \tau}\right),
$$

where $\gamma(T)=\gamma\left(T_{\mathrm{m}}\right)+\gamma_{T} \cdot\left(T-T_{\mathrm{m}}\right)$ is the temperature dependent surface tension coefficient above the melting temperature $T_{\mathrm{m}} ; K$ is the local mean curvature of the surface, and the subscripts ' $\mathrm{n}$ ', ' $\tau$ ' correspond to projections onto $\mathbf{e}_{\mathrm{n}}$ and $\mathbf{e}_{\tau}-$ the normal and tangent unit vectors at the free surface. In addition to the dynamic conditions (4), the calculated material fluid velocity $\mathbf{v}(t)$ moves continuously with the interface position $\mathbf{R}(t)$ :

$$
\mathbf{e}_{\mathrm{n}} \cdot \mathbf{v}=\mathbf{e}_{\mathrm{n}} \cdot \partial_{t} \mathbf{R}
$$

giving the instantaneous droplet shape. The thermal boundary conditions are the radiation loss on the surface of the levitated droplet:

$$
-\mathbf{n}(-k \nabla T)=\epsilon \sigma\left(T_{\mathrm{amb}}^{4}-T^{4}\right) .
$$

SPHINX uses the $k$ - $\omega$ turbulence model [12], which allows the investigation 
of the effect of the magnetic field on turbulence [11]:

$$
\begin{aligned}
& \partial_{t} k+\mathbf{v} \cdot \nabla k=\nabla \cdot\left[\left(\nu+\sigma_{k} \nu_{T}\right) \nabla k\right]+G-\beta^{*} \omega k-2 \alpha_{\mathrm{m}} k \frac{\sigma B^{2}}{\rho}, \\
& \partial_{t} \omega+\mathbf{v} \cdot \nabla \omega=\nabla \cdot\left[\left(\nu+\sigma_{\omega} \nu_{T}\right) \nabla \omega\right]+\alpha \frac{\omega}{k} G-\beta^{*} \omega^{2}-\alpha_{\mathrm{m}} \omega \frac{\sigma B^{2}}{\rho},
\end{aligned}
$$

with the boundary conditions at the free surface:

$$
\partial_{\mathrm{n}} k=0, \quad \partial_{\mathrm{n}} \omega=0 .
$$

Equations (1)-(8) are solved numerically using continuous transformation functions to account for shape change in the physical space. A spectral-collocation method is used to solve the resulting equations, according to which the velocity vector components and pressure are represented as series of Chebyshev polynomials and Legendre functions [9].

The electromagnetic force is computed using the integral equation representation for the axisymmetric shape of the metal, coil and the effective representation of the cold crucible wall. The electrical current distribution is given by the magnetic vector potential $\mathbf{A}$, the electric potential $\varphi$ and the motion induced part $\mathbf{v} \times \mathbf{B}$ :

$$
\mathbf{J}=\sigma\left(-\partial_{t} \mathbf{A}-\nabla \varphi+\mathbf{v} \times \mathbf{B}\right),
$$

where the magnetic field is

$$
\mathbf{B}=\nabla \times \mathbf{A},
$$

On the other hand, the electrical current distribution is related to the magnetic field and the vector potential $\mathbf{A}$ by the Biot-Savart law:

$$
\mathbf{A}(\mathbf{r})=\frac{1}{4 \pi} \iiint_{V(\mathbf{j} \neq 0)} \frac{\mathbf{j}\left(\mathbf{r}^{\prime}\right)}{\mathbf{r}-\mathbf{r}^{\prime}} \mathrm{d} \mathbf{r}^{\prime},
$$

which permits to solve the electrodynamic equations efficiently in the axisymmetric case of harmonic fields [13]:

$$
\mathbf{A}(\mathbf{r}, t)=\Re \mathrm{e}\left\{\mathbf{A}_{\mathrm{c}}(\mathbf{r}) \mathrm{e}^{i \omega t}\right\} .
$$

The motion-induced contribution $\mathbf{v} \times \mathbf{B}$ gives the time average (over the AC period) contribution to the electromagnetic force components expressed in spherical coordinates:

$$
\begin{aligned}
f_{v R} & =\sigma\left(-u\left\langle B_{\theta}^{2}\right\rangle-v\left\langle B_{R} B_{\theta}\right\rangle\right) \\
f_{v \theta} & =\sigma\left(u\left\langle B_{R} B_{\theta}\right\rangle-v\left\langle B_{R}^{2}\right\rangle\right) .
\end{aligned}
$$

COMSOL model results and comparison to SPHINX. COMSOL was used to predict the 3D electromagnetic field in a crucible consisting of a three-turn coil, sixteen copper segments (or 'fingers') and a hemi-spherical titanium charge of fixed geometry. The induced electrical current concentrates on the inner surfaces of the coil and is confined to a thin skin as indicated in Fig. 1. Current $\mathbf{J}$ penetrates to a greater depth in the titanium due to the lower electrical conductivity and larger skin depth. Allowing for some minor discrepancies, the 3D distribution of $|\mathbf{J}|$ in the charge produced with COMSOL (Fig. 2) was in good agreement with corresponding $2 \mathrm{D}$ results generated by SPHINX using the integral 


\section{Bojarevics, A.Roy, K.A.Pericleous}

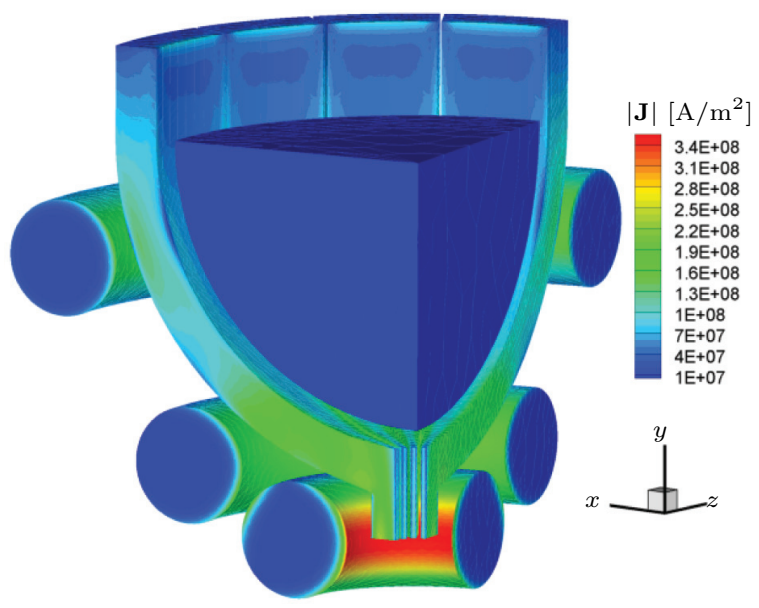

Fig. 1. One quarter of the crucible showing scalar plot of $|\mathbf{J}|$ on the external surfaces of the fingers and the coil computed with COMSOL; coil current $J_{\text {eff }}=5 \mathrm{kA}$ driven at $f=2 \mathrm{kHz}$.

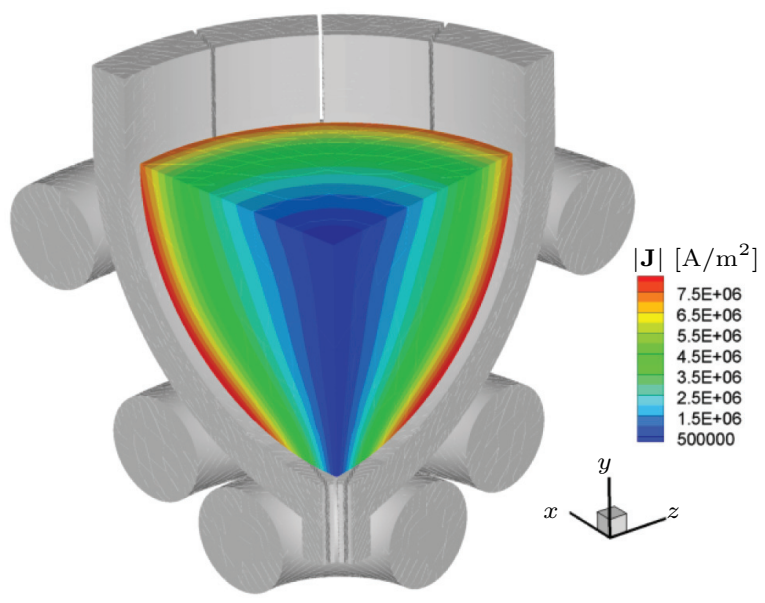

Fig. 2. One quarter of the crucible showing $|\mathbf{J}|$ in the titanium computed with COMSOL; coil current $J_{\text {eff }}=5 \mathrm{kA}$ driven at $f=2 \mathrm{kHz}$.

equation representation (Fig. 3). Hence, it can be concluded that the averaged axisymmetric approach implemented in SPHINX is sufficiently accurate to represent the effect of the full 3-dimensional electromagnetic field in the titanium, which is the main region of interest for this modelling. While it is not indicated, the maximum amplitude of magnetic flux density is about $0.122 \mathrm{~T}$ within the liquid metal electromagnetic skin-layer.

To model the crucible, we exploit periodicity of the field and consider an appropriate 3D wedge of the geometry consisting of half a segment and half a gap. A Neumann symmetry condition is imposed on the mid-plane of each finger, which forces streamlines of the induced current density to meet this plane at right-angles. Fig. 4 shows 3D streamlines of magnetic flux passing through the air gap between two adjacent copper wall segments. Some flux penetrates the segments, but it is confined to a thin skin-layer. In the titanium charge, the lines are distributed 


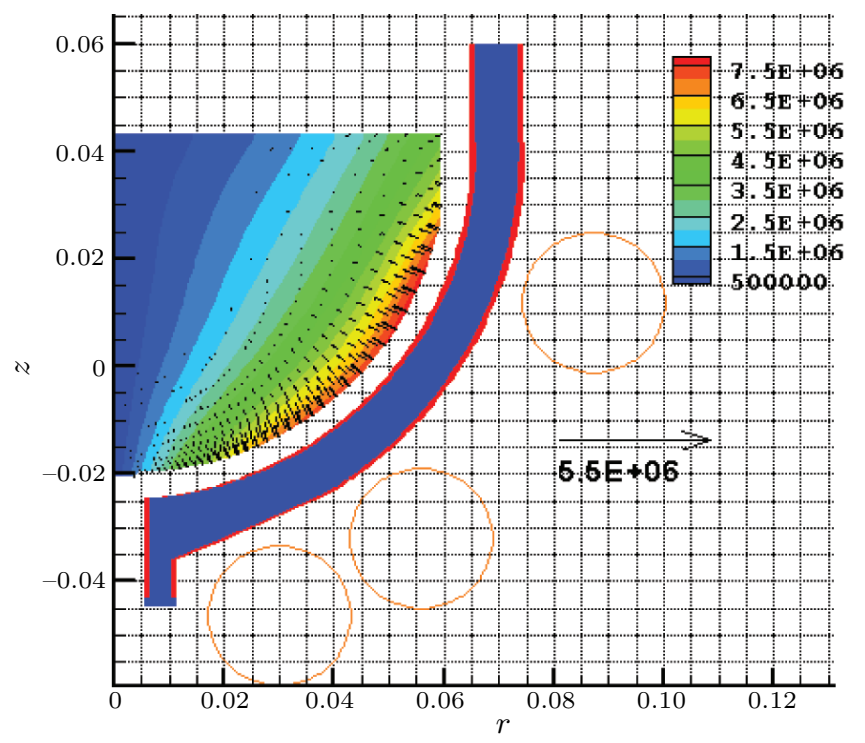

Fig. 3. Axisymmetric solution showing $|\mathbf{J}|=J_{\text {eff }}$ and the electromagnetic force distribution in the titanium computed by the SPHINX model; coil current $J_{\text {eff }}=5 \mathrm{kA}$ driven at $f=2 \mathrm{kHz}$.

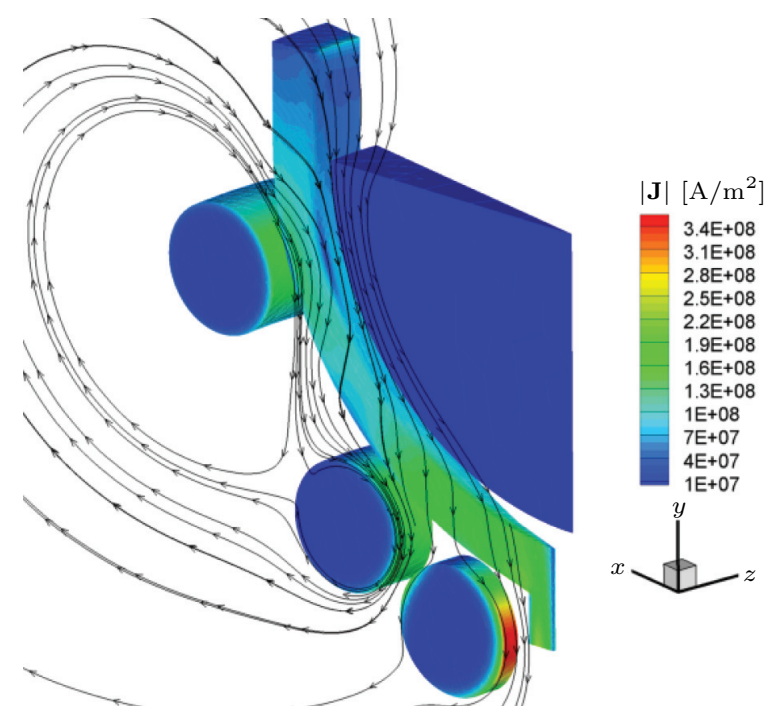

Fig. 4. Model showing (i) 3D streamlines of magnetic flux squeezing through the space between the fingers and (ii) scalar plot of $|\mathbf{J}|$ with $J_{\text {eff }}=5 \mathrm{kA}$ and $f=2 \mathrm{kHz}$ (due to symmetry only half a finger/air gap is considered).

more uniformly along the azimuthal direction. The relatively low frequency $(2 \mathrm{kHz})$ used in this example permits visualisation of the field within the copper segments. The circulating eddy currents are indicated by streamlines of $\mathbf{J}$ on various axial slices (Fig. 5). The magnitude of $|\mathbf{J}|$ is indicated by the colour flooding. Plots of the $J_{z}$-component (not shown) suggest that very little current flows axially in the segments, but this is thought to depend on the particular arrangement of the 


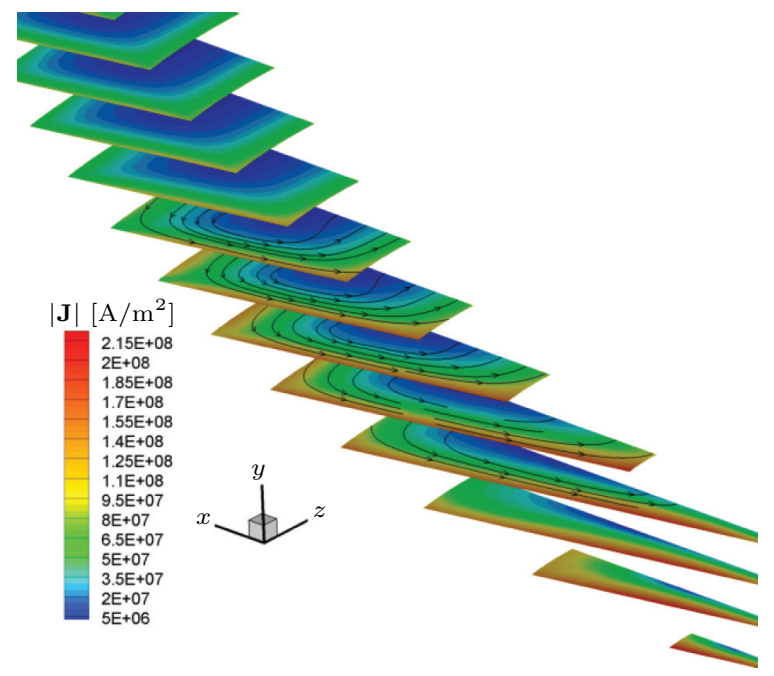

Fig. 5. Fig. 5. Scalar plot of $|\mathbf{J}|$ on various axial slices within half a finger due to circulating eddy currents; coil current $J_{\text {eff }}=5 \mathrm{kA}$ driven at $f=2 \mathrm{kHz}$.
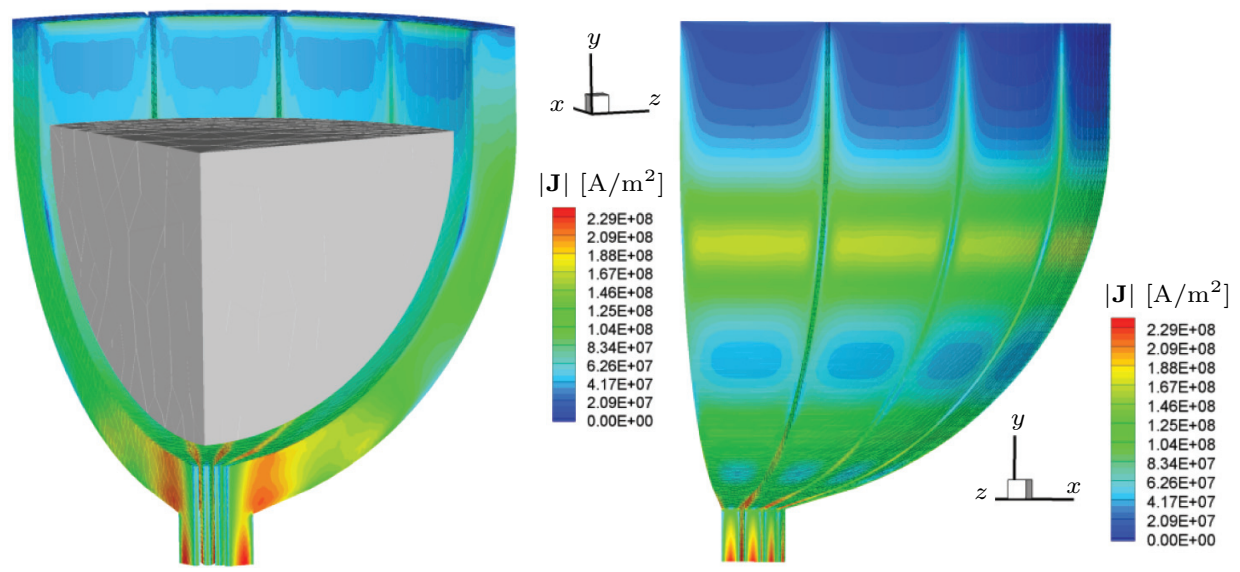

Fig. 6. Fig. 6. One quarter of the crucible showing $|\mathbf{J}|$ on the surface of the cold crucible segments computed by the $3 \mathrm{D}$ COMSOL model; coil current $J_{\text {eff }}=5 \mathrm{kA}$ driven at $f=2 \mathrm{kHz}$.

geometry. The magnitude of the induced current density is rather non-uniform over the external surfaces of the copper fingers, as seen from Fig. 6 in two different view positions. This reflects the position of the coils. Higher values of $|\mathbf{J}|$ occur due to localised confinement of flux flowing between the coils and the finger.

Further insight into the quantitative behaviour of the electromagnetic integral characteristics is obtained by inspecting the total Joule heating in different conducting regions of the model. Fig. 7 shows how power dissipation depends on frequency and compares the 3D model (which includes the finger) with the $2 \mathrm{D}$ model (no fingers). The results indicate that Joule heating within the liquid titanium and the copper coil over the range of frequencies considered is largely unaffected by the presence of the finger segments. A very similar conclusion is apparent from Fig. 8, which shows the total force in the vertical direction acting 


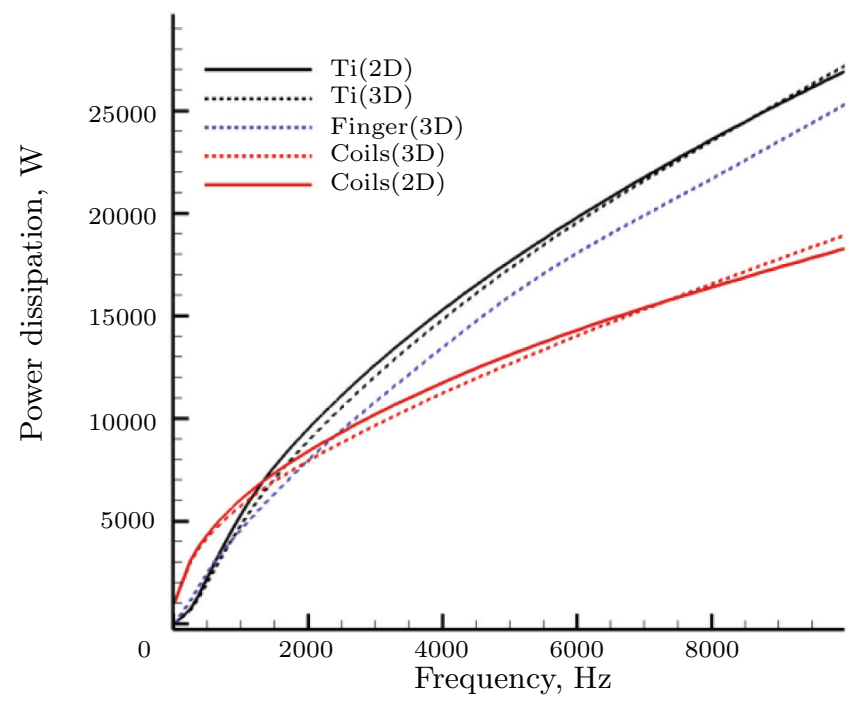

Fig. 7. Power dissipation in conducting regions as a function of frequency when $J_{\text {eff }}=5 \mathrm{kA}$.

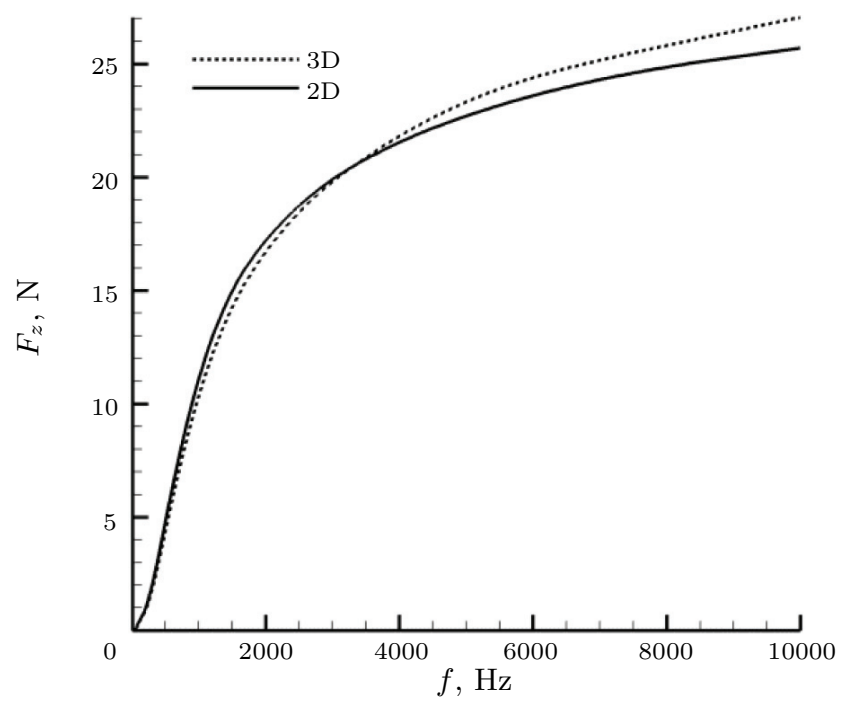

Fig. 8. Time-averaged axial force on the titanium as a function of frequency with $J_{\text {eff }}=5 \mathrm{kA}$.

on the titanium load. As the frequency increases, the force will asymptotically approach a constant value, which however is not attained for the rather moderate values accessible with the present 3D COMSOL finite element solution.

The $3 \mathrm{D}$ effect of the fingers is very important when considering the total power requirements of the electromagnetic system. The internally circulating induced electrical currents in the fingers account for a significant part of the total electrical energy consumption, as demonstrated in Fig. 9. The efficiency of the cold crucible is estimated as the ratio of the useful energy $Q_{\mathrm{Ti}}$ (used to heat the titanium load) to the total electrical power dissipation $Q_{\text {tot }}$ in the whole system. The efficiency for both $2 \mathrm{D}$ and $3 \mathrm{D}$ cases reaches a saturation level at higher frequencies. In 


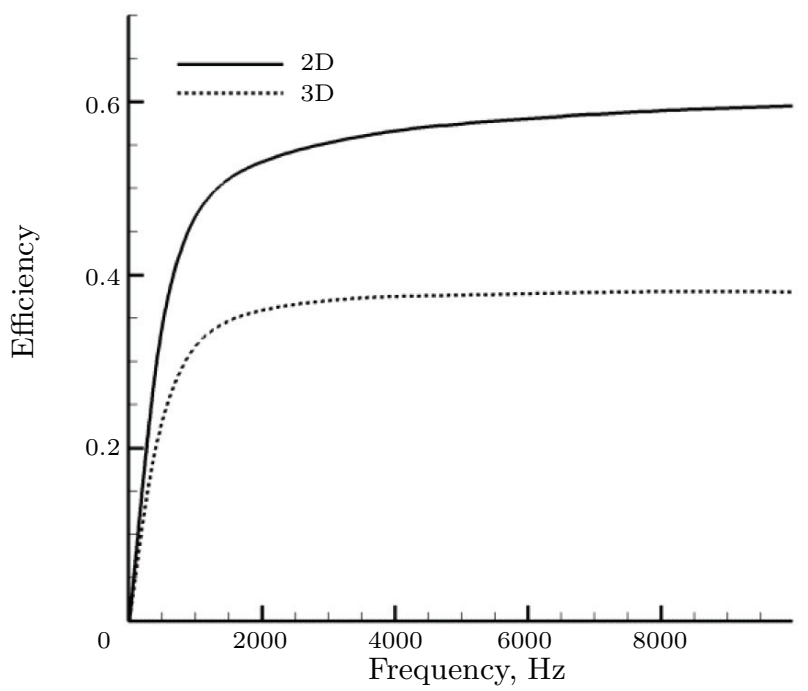

Fig. 9. Efficiency $\left(Q_{\mathrm{Ti}} / Q_{\text {tot }}\right)$ as a function of frequency $(\mathrm{Hz})$; "3D" = model consisting of coils, titanium and 16 fingers; "2D" = axisymmetric model consisting of titanium and coils.

particular, by comparing the $3 \mathrm{D}$ and $2 \mathrm{D}$ results we see that the presence of the finger segments greatly reduces the energy efficiency.

SPHINX results for the melt dynamics. The axisymmetric approximation is a reasonable choice for the liquid metal region, as we have seen from the results of the previous section. The integral equation formulation used in the SPHINX code avoids the need to mesh the surrounding air volume; instead, the mesh used for fluid dynamics can be directly used for electromagnetic field calculations in the fluid. The additional current carrying regions (coil and the cold crucible wall) can be approximated as axisymmetric using the effective azimuthal current representation as described in previous publications [2, 13]. The validity of this approach is supported by the COMSOL 3D simulations.

The SPHINX model follows the dynamic development of the liquid surface supported by the magnetic force and the associated velocity field. The final position of the liquid metal surface is determined by the complex interaction of the induced electromagnetic fields in various regions and the fluid dynamic effects. This is a complex dynamic process, where various properties are important, not least the internal fluid flow turbulence with the magnetic interaction.

There is a pronounced dependence of the flow intensity on the applied AC field frequency because of the change in electromagnetic field penetration depth. The cases considered in the previous section are limited to $2-10 \mathrm{kHz}$ by the ability of the 3D finite element meshing to accurately resolve the skin effect in the conducting domains. A higher frequency AC field is preferable because of the lower penetration to the liquid domain, concentrating the magnetic confinement force near the surface and stabilising it. Therefore, levitation of a large fluid mass needs a high frequency solution. The following solutions for stably levitated liquid metal were run using a higher $\mathrm{AC}$ frequency of $20 \mathrm{kHz}$.

Fig. 10 demonstrates the electromagnetic solution at a time just after preheating and completely melting the hemi-spherically-shaped liquid metal zone. The electromagnetic force is concentrated in a thin skin-layer, and is variable along the boundary. The computed estimates give $70 \mathrm{~kW}$ of the power dissipated in the 


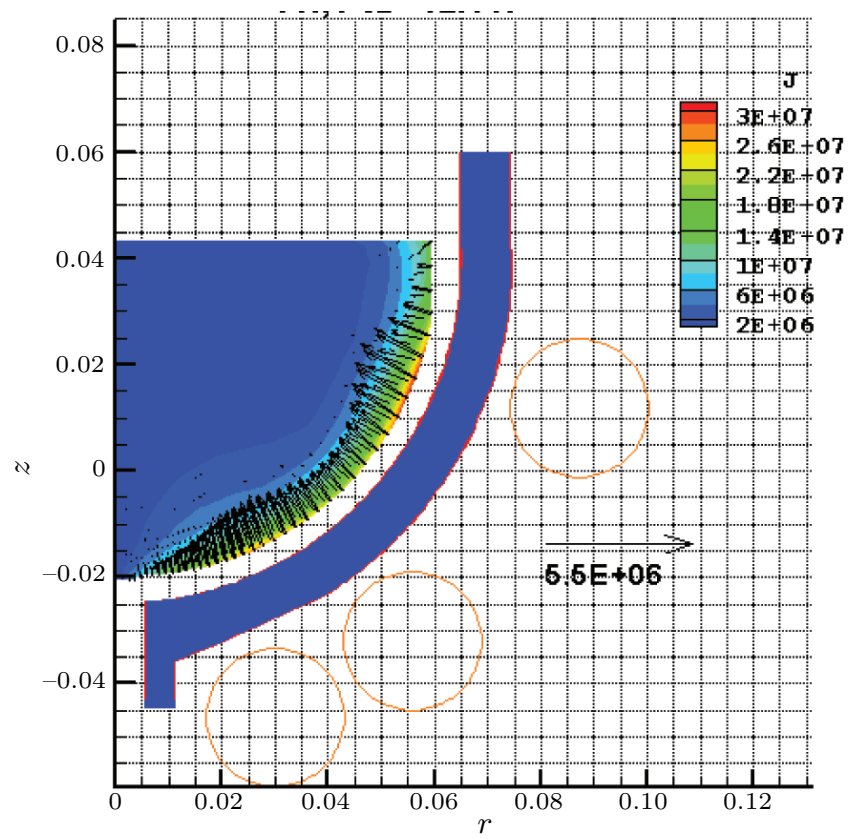

Fig. 10. Axisymmetric solution showing $|\mathbf{J}|=J_{\text {eff }}$ and the electromagnetic force distribution in the hemi-spherical titanium at initial time moment computed by the SPHINX model; coil current $J_{\text {eff }}=5 \mathrm{kA}$ driven at $f=20 \mathrm{kHz}$.

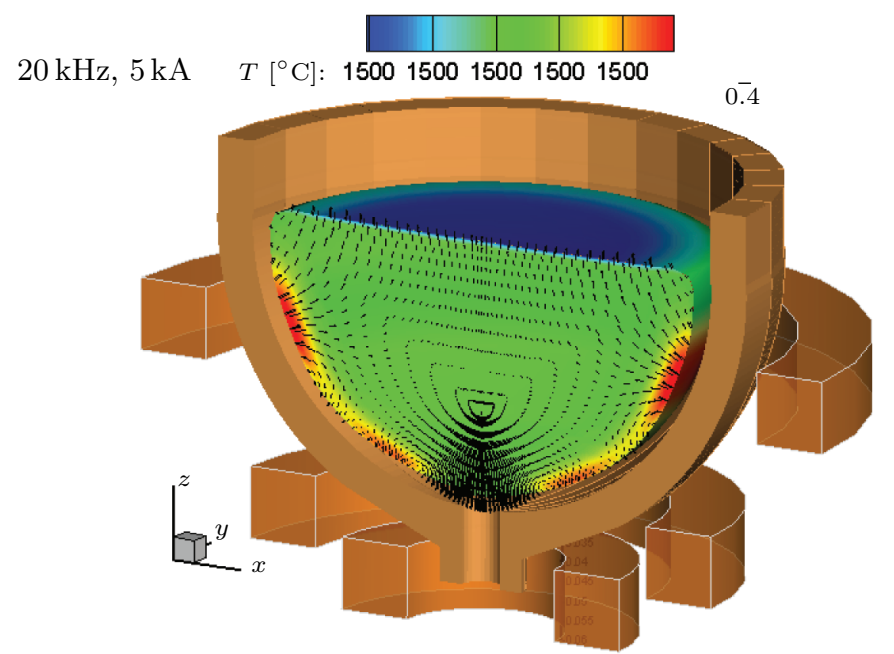

Fig. 11. The initial velocity field and temperature distribution in the molten hemispherical titanium computed by the SPHINX model.

cold crucible walls (copper segments) and $53 \mathrm{~kW}$ of Joule heating for the liquid titanium. The computed total electromagnetic force in the vertical direction on the whole volume of the liquid metal is about $42.1 \mathrm{~N}$, which well exceeds the total weight $20.4 \mathrm{~N}$ of the metal load. However, the force distribution is such that the rotor of the local electromagnetic force rot $\mathbf{f}_{\mathrm{e}}$ drives an intense fluid flow, the liquid interface moves and the electromagnetic force distribution changes in time. Fig. 11 shows the velocity field and the temperature distribution at an early time when the surface of the liquid metal is still hemi-spherical. 


\section{Bojarevics, A.Roy, K.A.Pericleous}

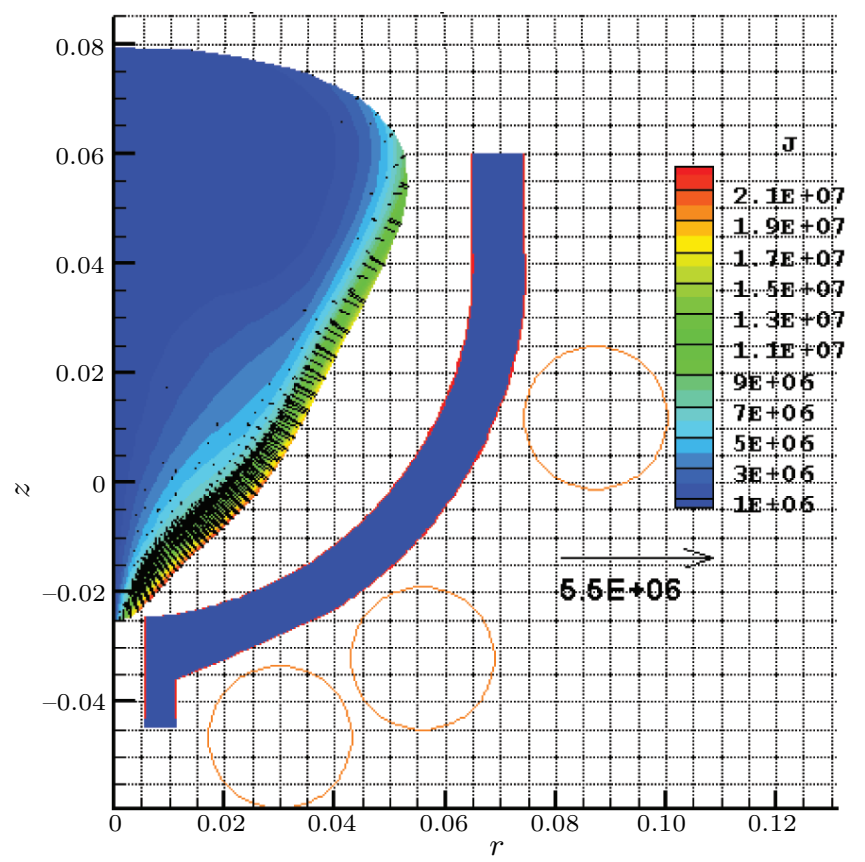

Fig. 12. Axisymmetric solution showing $|\mathbf{J}|=J_{\text {eff }}$ and the electromagnetic force distribution in the magnetically levitated liquid titanium after several seconds of the flow and shape adjustment to a quasi-stationary shape computed by the SPHINX model; coil current $J_{\text {eff }}=5 \mathrm{kA}$ driven at $f=20 \mathrm{kHz}$.

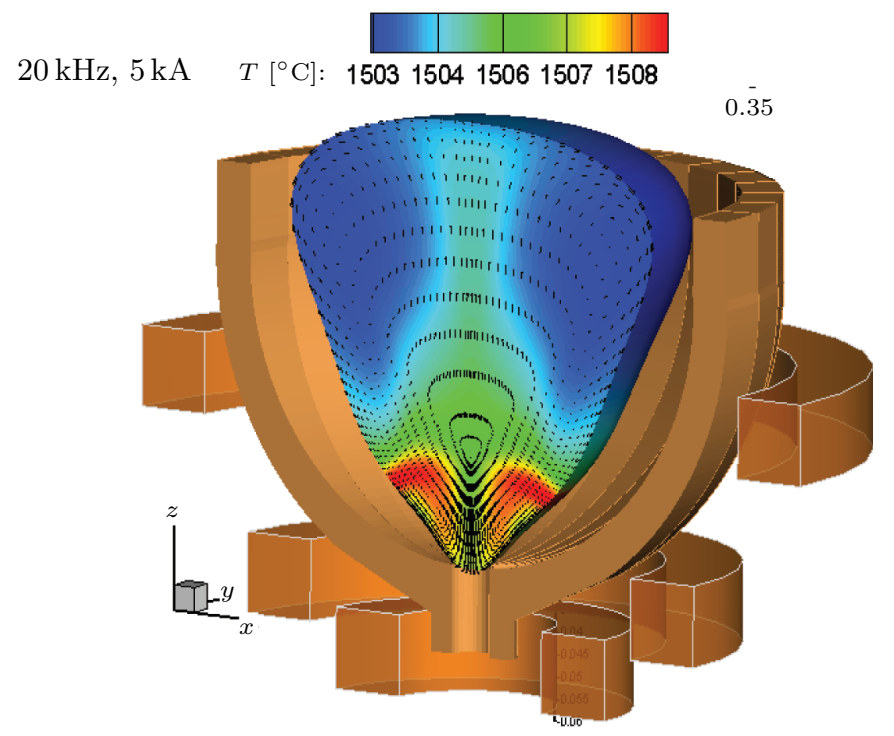

Fig. 13. The velocity field and temperature distribution in the magnetically levitated liquid titanium after several seconds of the flow and shape adjustment to a quasistationary shape computed by the SPHINX model.

After several seconds of intense flow development, computed numerically in adjusting second order accurate implicit time stepping $(\triangle t \leq 0.0005 \mathrm{~s})[2,13]$, the interface moves to the relatively stable position shown in Figs. 12 and 13, which show the electromagnetic and velocity field representations, respectively. 


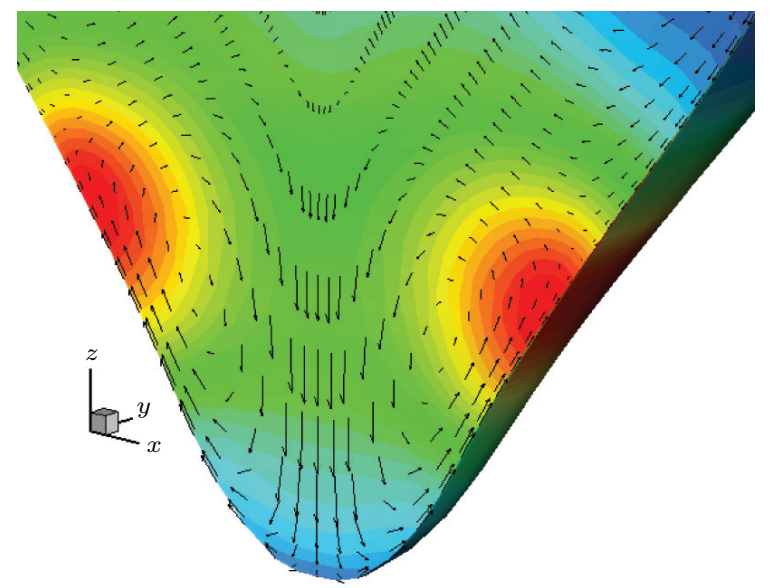

Fig. 14. The zoom-in to the velocity field at the bottom part of the magnetically levitated liquid titanium in a quasi-stationary shape.

The total electromagnetic force, acting on the fluid volume drops significantly, approaches in an oscillating way the value of the total weight of the liquid metal. The total Joule heating decreases significantly compared to what was achieved with the initial hemi-spherical profile due to the surface being pushed away from the container walls.

During the surface and flow adjustment process, the liquid top surface performs several oscillations. The bottom initially flows down to reach the contact to the solid wall, but then is pushed up, detaches and, after several oscillations, assumes the quasi-steady levitated position. The zoom-in view for the bottom velocity field at a particular time is shown in Fig. 14. The bottom shape is slightly oscillating, which constantly readjusts the electromagnetic, velocity and temperature field. However, on a larger scale the liquid appears to be levitated completely, being supported by the magnetic forces only.

The mechanism of the levitation and, particularly, the magnetic support at the bottom appears to be dynamic in nature. The electromagnetic force is zero at the bottom tip position (see Fig. 12) and the surface tension effect is clearly not sufficient to support the $2 \mathrm{~kg}$ of liquid titanium in this rather rounded shape of relatively large curvature radius. The explanation for the fact that the liquid at the bottom is prevented from leaking and flowing down is related to the particular velocity field in this region. The bottom vortex in Fig. 14 is maintained by the rotational nature of the electromagnetic force $\left(\operatorname{rot} \mathbf{f}_{\mathrm{e}} \neq 0\right)$, which drives the fluid tangentially upwards at the side surface of the liquid, away from the bottom stagnation point. Due to the continuity $(\operatorname{div} \mathbf{v}=0)$ of the velocity field, the outflow at the bottom is redirected to the intense flow upwards along the side surface. The final appearance is rather smooth, but during the detachment process, there are quite abrupt changes in velocity field topology, requiring dynamic time step adjustments to account for the curvature change and surface tension, see the boundary condition (4).

To achieve the full levitation of the large fluid mass, rather elaborate optimization of the bottom flow field is required. This involves multiple numerical test runs and adjustment of the coil position until the desired force field is created to ensure the dynamic balance at the bottom. An example of an "unsuccessful" 


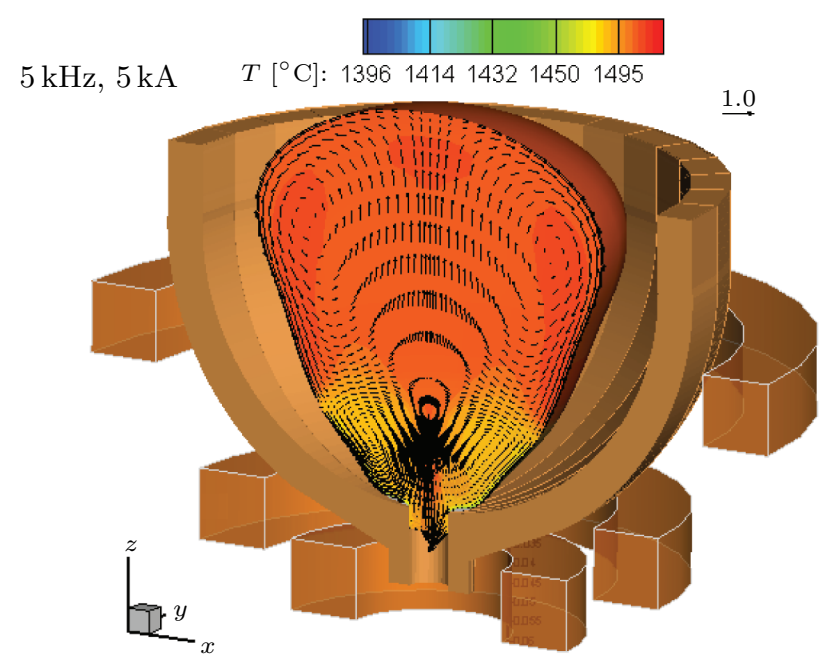

Fig. 15. The velocity field and temperature distribution in the magnetically confined liquid titanium leaking at the bottom hole of the crucible after several seconds of the flow and shape adjustment to a quasi-stationary shape.

levitation experiment is shown in Fig. 15, where the liquid metal is flowing out at the bottom hole of the cold crucible. This example, which was run at $5 \mathrm{kHz}$, also demonstrates the difficulty to achieve levitation at lower coil excitation frequencies. The typical velocities are about $1 \mathrm{~m} / \mathrm{s}$ for the $5 \mathrm{kHz}$ case if compared to $0.35 \mathrm{~m} / \mathrm{s}$ for the $20 \mathrm{kHz}$ case (Fig. 13).

1. Conclusions. Two independent numerical codes are used to model the $\mathrm{AC}$ magnetic levitation of a large mass of liquid. The full 3D simulation using COMSOL shows that the field seen by the liquid metal is approximately axisymmetric and largely unaffected by the presence of the finger segments. While this is true, the presence of the fingers significantly reduces the energy efficiency. The integral equation approach in the SPHINX code permits high frequency solutions at dynamically adjusted surface positions. Full levitation of the liquid metal is achievable, but requires a careful optimisation of the electromagnetic force for generating tangential flow along the surface away from the bottom stagnation point. The bottom confinement is dynamic in nature.

\section{REFERENCES}

[1] I. Egry, A. Diefenbach, W. Dreier, J. Piller. Containerless processing in space - thermophysical property measurements using electromagnetic levitation. Int. J. Thermophys., vol. 22 (2001), pp. 569-578.

[2] V. Bojarevics, R.A. Harding, K. Pericleous and M. Wickins. The development and validation of a numerical model of an induction skull melting furnace. Metallurgical and Materials Transactions, vol. 35B (2004), pp. 785803.

[3] L. Baptiste, N. van Landschoot, G. Gleijm, J. Priede, J. SchaDE VAN Westrum, H. Velthuis, T.-Y. Kim. Electromagnetic levitation: A new technology for high rate physical vapour deposition of coatings onto metallic strip. Surface 83 Coatings Technology, vol. 202 (2007), pp. 1189-1193. 
[4] P. Gillon. Synthesis of bulk metallic glass forming alloys in a cold crucible induction furnace. Proc. the 4th Int. Symp. Electromagn. Processing Materials (Lyon, France, 2003), pp. 220-225.

[5] T. Tanaka, N. Yoshida, Y. Ikenaga, M. Horie. High volume reduction and group Separation of simulated fission products by a cold crucible. Proc. the 3rd Int. Symp. Electromagn. Processing Materials, ISIJ (Nagoya, Japan, 2000), pp. 271-276.

[6] T. Toh, H. Yamamura, H. Kondo, M. Wakoh, Sh. Shimasaki S. TaniGUCHI. Kinetics evaluation of inclusions removal during levitation melting of steel in a cold crucible. ISIJ International, vol. 47 (2007), no. 11, pp. 16251632 .

[7] H. Tadano, M. Fujita, T. Take, K. Nagamatsu, A. Fukuzawa. Levitational melting of several kilograms of metal with a cold crucible. IEEE Trans. Magn., vol. 30 (1994), no. 6, pp. 4740-4742.

[8] T. Okumura, K. Yamamoto, M. Shibata. Large scale cold crucible levitation melting furnace with a bottom tapping nozzle. Proc. The 6th Internat. Conf. Electromagnetic Processing Materials (Dresden, Germany, 2009), pp. 521-524.

[9] V. Bojarevics, K. Pericleous. Modelling electromagnetically levitated liquid droplet oscillations. ISIJ International, vol. 43 (2003), no. 6, pp. 890 898.

[10] R.A. Harding， M. Wickins， G. Keough， K. Pericleous， V. BojareVICS. The use of combined DC and AC fields to increase superheat in an induction skull melting furnace. Proc. the 2005 Int. Symp. Liquid Metal Processing and Casting (Santa Fe, USA, 2005), pp. 201-210.

[11] O. WidLund. Modelling of magnetohydrodynamic turbulence (Ph.D. Thesis, Royal Institute of Technology, Stockholm, Sweden, 2000).

[12] D.C. Wilcox.. Turbulence modelling for CFD (2nd ed., DCW Industries, California, 1998).

[13] V. Bojarevics, K. Pericleous, M. Cross. Modelling the dynamics of magnetic semi-levitation melting. Metall. Materials Trans., vol. 31B (2000), pp. $179-189$. 